\title{
Non-monogamy of Quantum Discord and Upper Bounds for Quantum Correlation
}

\author{
Xi-Jun Ren* \\ School of Physics and Electronics, Henan University, Kaifeng 475001, China \\ Heng Fan' \\ Beijing National Laboratory for Condensed Matter Physics, \\ Institute of Physics, Chinese Academy of Sciences, Beijing 100190, China
}

\begin{abstract}
We consider a monogamy inequality of quantum discord in a pure tripartite state and show that it is equivalent to an inequality between quantum mutual information and entanglement of formation of two parties. Since this inequality does not hold for arbitrary bipartite states, quantum discord can generally be both monogamous and polygamous. We also carry out numerical calculations for some special states. The upper bounds of quantum discord and classical correlation are also discussed and we give physical analysis on the invalidness of a previous conjectured upper bound of quantum correlation. Our results provide new insights for further understanding of distributions of quantum correlations.
\end{abstract}

PACS numbers: 03.65.Ud, 03.65.-w

\section{INTRODUCTION}

Quantum states possess quantum correlations which are classically unobtainable and act as an invaluable resource for quantum information processing. For a long time, interests on quantum correlations focused on quantum entanglement which is a special kind of quantum correlation enabling fascinating tasks such as quantum key distribution, quantum teleportation and superdense coding, etc [1]. Quantum entanglement does not exist in separable states which are mixtures of separable direct product states [2]. However, recent researches show that some separable quantum states can exhibit their quantumness in many interesting circumstances. In Ref. [3], Knill and Laflamme introduced an interesting computation model, deterministic quantum computation with one quantum bit (DQC1), for which unentangled states can provide exponential speed up over the best known classical algorithms. Together with some other interesting tasks, such as locking of large amount of classical correlations with small classical communication in unentangled states, they ignite interests and studies on more general nonclassical correlations or quantumness of quantum states [4-10]. From an extensive background, the nonclassical correlations or quantumness of quantum states are always of fundamental importance for both quantum information theory and quantum mechanics .

Among the nonclassical correlation measures proposed with different motivations, quantum discord is an important one for capturing all the nonclassical correlations in a bipartite quantum state [4, 5]. Researches on quantum discord develop quickly in recent years. Direct calculations were carried out for some interesting quantum states [11, 12]. Operational meanings of quantum discord

\footnotetext{
*Electronic address: renxijun@mail.ustc.edu.cn
}

${ }^{\dagger}$ Electronic address: hfan@iphy.ac.cn were given in terms of some other important concepts like quantum state merging $9,14,15 \mid$. The dynamics of quantum discord were discussed in [16, 17]. Especially, experiments for quantum discord were also carried out [16, 18]. In this paper, we are mainly concerned with the monogamy property of quantum discord.

Unlike the arbitrary shareability of classical correlations among multipartite systems, the shareability of quantum correlations is always constrained by some monogamy relation as in case of entanglement measure [19]. It says that for a multipartite state $\rho_{A_{0} A_{1} \ldots A_{n}}$ and a quantum correlation measure $E$, the quantum correlation between $A_{0}$ and $A_{1}, A_{2}, \ldots, A_{n}$ as a whole should be larger than the sum of correlations between $A_{0}$ and $A_{1}$, $A_{2}, \ldots, A_{n}$ separately, i. e. $E_{A_{0} \mid A_{1} \ldots A_{n}} \geq \sum_{i} E_{A_{0} A_{i}}$. The underlying intuition is that their difference should be genuine multipartite quantum correlations which may exist only among three or more parties. In [19, 20], the authors constructed the monogamy relation for qubit systems and concurrence which is a entanglement measure first introduced by Hill and Wootters [21]. The monogamy relation in continuous systems was provided in [22]. More discussions on monogamy of different quantum correlation measures can be found in, for example, Refs. 23 26]. Since quantum discord quantifies the quantum correlations in a bipartite state, it is interesting to study whether it also respects monogamy relation. Recently, Prabhu et al. 27] and Giorgi [28] have studied the following monogamy relation for quantum discord,

$$
D^{\leftarrow}\left(\rho_{A B}\right)+D^{\leftarrow}\left(\rho_{A C}\right) \leq D^{\leftarrow}\left(\rho_{A \mid B C}\right) .
$$

They showed that such a monogamy relation generally does not hold. In this paper, we will study a different kind of monogamy relation for quantum discord,

$$
D^{\rightarrow}\left(\rho_{A B}\right)+D^{\rightarrow}\left(\rho_{A C}\right) \leq D^{\rightarrow}\left(\rho_{A \mid B C}\right),
$$

for a pure tripartite state $\left|\Psi_{A B C}\right\rangle$. Because of the asymmetry of quantum discord, the above two monogamy relations are quite different. Physically, the inequality (1) 
means that the measurement is taken on two parties, $B$ and $C$, coherently in right hand side of the inequality and individually in left hand side of the inequality. However, the inequality (2) means that only one local measurement on party $A$ is performed.

The outline of this paper is as follows. In the following section, after reviewing the definition of quantum discord, we derive an equivalent relation to the monogamy inequality (2). Then through concrete examples we numerically show that the monogamy relation (2) does not generally hold. With squashed entanglement we also provide a special case when monogamy relation (2) does hold. In the third section, we discuss another interesting issue on quantum discord, the upper bounds of quantum and classical correlations. Here, we give physical explanations on the invalidity of previous conjectured upper bound of quantum correlations. Finally we give our conclusions.

\section{MONOGAMY RELATIONS OF QUANTUM DISCORD IN A PURE TRIPARTITE STATE}

In this section we will point out that the monogamy inequality (2) can be reduced to a relation between entanglement of formation (EoF) [29], a well-accepted entanglement measure, and quantum mutual information. Before expanding our discussions, we first review the definition of quantum discord. The definition is based on the difference between the total correlation and the classical correlation in the state, quantified by quantum mutual information and quantum conditional entropy by a local measurement, respectively. For a general bipartite state $\rho_{A B}$, quantum mutual information $I\left(\rho_{A B}\right)=S\left(\rho_{A}\right)+S\left(\rho_{B}\right)-S\left(\rho_{A B}\right)$, is generally taken to be the measure of total correlations, both classical and quantum. In order to quantify the classical correlation, a positive operator valued measurement (POVM) $\left\{\Pi_{i}\right\}$ is made on party $A$, the resulting state given by the shared ensemble $\left\{p_{i}, \rho_{B \mid i}\right\}$, where $p_{i}=\operatorname{Tr}_{A, B}\left(\Pi_{i} \rho_{A B}\right), \rho_{B \mid i}=\operatorname{Tr}_{A}\left(\Pi_{i} \rho_{A B}\right) / p_{i}$. Similar to the classical conditional entropy, quantum conditional entropy is defined as $S_{\left\{\Pi_{i}\right\}}(B \mid A)=\sum_{i} p_{i} S\left(\rho_{B \mid i}\right)$, then an alternative version of quantum mutual information with respect to POVM $\left\{\Pi_{i}\right\}$ is defined as $J_{\left\{\Pi_{i}\right\}}\left(\rho_{A B}\right)=$ $S\left(\rho_{B}\right)-S_{\left\{\Pi_{i}\right\}}(B \mid A)$. Maximizing $J_{\left\{\Pi_{i}\right\}}\left(\rho_{A B}\right)$ over all POVMs $\left\{\Pi_{i}\right\}$, we arrive at a measurement independent quantity $J \rightarrow\left(\rho_{A B}\right)=\max _{\left\{\Pi_{i}\right\}}\left[S\left(\rho_{B}\right)-S_{\left\{\Pi_{i}\right\}}(B \mid A)\right]$ which captures all the classical correlation present in $\rho_{A B}$. Taking the difference between total correlations and classical correlation, we obtain the following one way quantum discord,

$$
\begin{aligned}
D^{\rightarrow}\left(\rho_{A B}\right) & =I\left(\rho_{A B}\right)-J^{\rightarrow}\left(\rho_{A B}\right) \\
& =S\left(\rho_{A}\right)-S\left(\rho_{A B}\right)+\min _{\left\{\Pi_{i}\right\}} \sum_{i} p_{i} S\left(\rho_{B \mid i}\right) .(3)
\end{aligned}
$$

Symbol $\rightarrow$ shows that such defined correlation measure is asymmetric, i.e. generally $D^{\rightarrow}\left(\rho_{A B}\right) \neq D^{\leftarrow}\left(\rho_{A B}\right)$, where
$D^{\leftarrow}\left(\rho_{A B}\right)$ is based on POVM on party $B$.

Now, let us consider a pure tripartite state $\left|\Psi_{A B C}\right\rangle$. The quantum discord between $A$ and $B C$ as a whole is the von Neumann entropy of $A$,

$$
D^{\rightarrow}\left(\rho_{A \mid B C}\right)=S\left(\rho_{A}\right)
$$

This means that by a von Neumann measurement with basis in agreement with the Schmidt decomposition of bipartite partition $\left|\Psi_{A \mid B C}\right\rangle$, the result is the quantum discord. On the other hand, for pure state $\left|\Psi_{A B C}\right\rangle$, we have the following relations between quantum discord and EoF [30],

$$
\begin{aligned}
& D^{\rightarrow}\left(\rho_{A B}\right)=S\left(\rho_{A}\right)-S\left(\rho_{A B}\right)+E_{F}\left(\rho_{B C}\right), \\
& D^{\rightarrow}\left(\rho_{A C}\right)=S\left(\rho_{A}\right)-S\left(\rho_{A C}\right)+E_{F}\left(\rho_{B C}\right) .
\end{aligned}
$$

From these relations, we have

$$
\begin{aligned}
& D^{\rightarrow}\left(\rho_{A \mid B C}\right)-D^{\rightarrow}\left(\rho_{A B}\right)-D^{\rightarrow}\left(\rho_{A C}\right) \\
= & S\left(\rho_{A B}\right)+S\left(\rho_{A C}\right)-S\left(\rho_{A}\right)-2 E_{F}\left(\rho_{B C}\right) \\
= & S\left(\rho_{B}\right)+S\left(\rho_{C}\right)-S\left(\rho_{B C}\right)-2 E_{F}\left(\rho_{B C}\right) \\
= & I\left(\rho_{B C}\right)-2 E_{F}\left(\rho_{B C}\right) .
\end{aligned}
$$

Therefore the monogamy relation (2) is reduced to

$$
E_{F}\left(\rho_{B C}\right) \leq \frac{I\left(\rho_{B C}\right)}{2}
$$

Inequality (6) shows that an inequality between quantum mutual information and EoF of a bipartite state implies the monogamy inequality of quantum discord in a tripartite state, which is the purification of the bipartite state. In [31], $I\left(\rho_{B C}\right)-2 E_{F}\left(\rho_{B C}\right)$ was also found to be equal to the difference of classical correlation $J \rightarrow\left(\rho_{A B}\right)$ and quantum discord $D^{\rightarrow}\left(\rho_{A B}\right)$ between $A B$ which was named discrepancy. We know that quantum mutual information is commonly considered to quantify the total correlations [32] and entanglement of formation is a measure of entanglement. Interestingly, for any pure bipartite state $\left|\Psi_{A B}\right\rangle$, we have that quantum mutual information, $I\left(\Psi_{A B}\right)=2 S\left(\rho_{A}\right)$, is two times of the entanglement of formation of state $\left|\Psi_{A B}\right\rangle$. This inequality seems reasonable. Actually inequality (6) has already been analyzed as a postulate for measures of quantum correlation in [33], where Li and Luo show that there are states for which inequality (6) does not hold and they also argue that EoF may not be a proper quantum correlation measure consistent with quantum mutual information. Here, the existence of states violating eq.(6) shows that monogamy relation (2) does not generally hold. In the following we will discuss monogamy relation (2) through some tripartite states.

Generalized pure three-qubit GHZ and $W$ states.-In Ref. [27], a necessary and sufficient condition for quantum discord being monogamous with inequality(1) is given and applied to generalized GHZ and W states. 


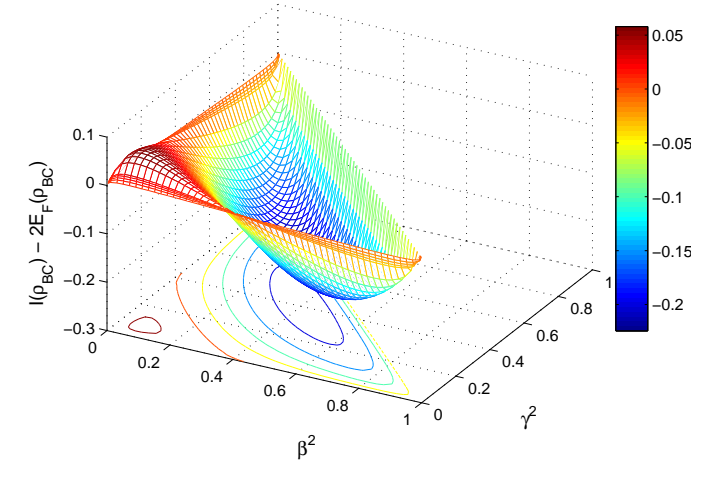

FIG. 1: Difference between mutual information and two times EoF for a bipartite state of parties $B$ and $C$ from a generalized $W$ state in Eq.(7). It can be found that quantum discord can be both monogamous and polygamous.

Based on numerical calculations of generalized W state, it was conjectured that the quantum discord in these states is polygamous which is confirmed in Ref. [28] with the conservation law for distributed EoF and quantum discord [30]. Here, concerning with our proposed monogamy inequality (2), it can be easily seen that for generalized GHZ states $\left|\Psi_{A B C}^{G H Z}\right\rangle=\alpha|000\rangle_{A B C}+\beta|111\rangle_{A B C}$, relation (6) holds since EoF is simply zero and hence monogamy relation (2) holds. However, generalized W states are different and direct calculations show that quantum discord in generalized W states can be both monogamous and polygamous with inequality (2). Explicitly, the generalized W states take the form,

$$
\left|\Psi_{A B C}^{W}\right\rangle=\alpha|011\rangle_{A B C}+\beta|101\rangle_{A B C}+\gamma|110\rangle_{A B C} .
$$

Without lose of generality, we assume that $\alpha, \beta, \gamma$ are all real, and the normalization condition $\alpha^{2}+\beta^{2}+\gamma^{2}=1$. We calculate $I\left(\rho_{B C}\right)-2 E_{F}\left(\rho_{B C}\right)$ for this state and the results are given in Fig.1. It can be easily seen that though in most cases quantum discord is polygamous $\left(D_{A \rightarrow B C}-D_{A \rightarrow B}-D_{A \rightarrow C}<0\right)$, when $\beta^{2}, \gamma^{2}$ are small we have $D_{A \rightarrow B C}-D_{A \rightarrow B}-D_{A \rightarrow C} \geq 0$, which means that quantum discord is monogamous between $A$ and $B, C$. In the reduced bipartite state $\rho_{B C}, \alpha^{2}$ is the proportion of direct product state $|11\rangle, \beta^{2}+\gamma^{2}$ quantifies the proportion of entangled state $\beta|01\rangle_{B C}+\gamma|10\rangle_{B C}$. Fig.1 implies that monogamy relation (2) is roughly related to the entangled proportion $\beta^{2}+\gamma^{2}$ shared between $B$ and $C$. Monogamy holds when this entangled proportion is small and polygamy holds when it is large. Similar analysis can be made on generalized GHZ class states which contain the above generalized GHZ states $\left|\Psi_{A B C}^{G H Z}\right\rangle$ as a subset. One simple example is, $\left|\Psi_{A B C}\right\rangle=\alpha|000\rangle_{A B C}+\beta|100\rangle_{A B C}+\gamma|111\rangle_{A B C}$, one can find that these states can also be both monogamous and polygamous.

Isotropic states and Werner states.- Since monogamy inequality (2) is equivalent to inequality (6) which concerns only about a bipartite state $\rho_{B C}$, while party $A$

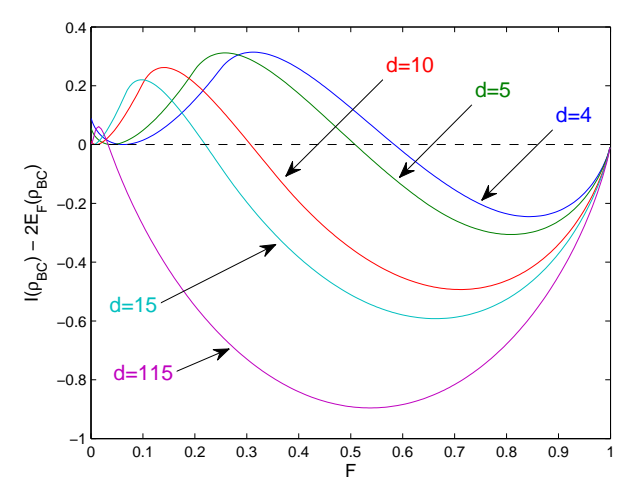

FIG. 2: Difference between mutual information and two times EoF for isotropic state in Eq. (8). When dimension $d$ increases, the region of negative becomes larger corresponding to polygamy for a pure tripartite state.

can be regarded as an extension of this bipartite state for purification, we need only to analyze bipartite state $\rho_{B C}$. In the following we will consider bipartite isotropic states [34] and Werner states [41] which have analytical expressions for EoF [34]. The isotropic states take the following form,

$$
\rho_{B C}=\frac{1-F}{d^{2}-1}\left(I-\left|\Psi^{+}\right\rangle\left\langle\Psi^{+}\right|\right)+F\left|\Psi^{+}\right\rangle\left\langle\Psi^{+}\right|
$$

where, $0 \leq F \leq 1$ and $\left|\Psi^{+}\right\rangle=\frac{1}{\sqrt{d}} \sum_{i=1}^{d}|i i\rangle, d$ is dimension of Hilbert space $B$ and $C$. First, since both reduced density matrices of $B, C$ are $I / d$, we have $S\left(\rho_{B}\right)=$ $S\left(\rho_{C}\right)=\log _{2} d$. Second, $S\left(\rho_{B C}\right)$ can be directly calculated as,

$$
\begin{aligned}
S\left(\rho_{B C}\right)= & -F \log _{2} F-(1-F) \log _{2}(1-F) \\
& +(1-F) \log _{2}\left(d^{2}-1\right) .
\end{aligned}
$$

From Ref.[34], we know that the FoF of $\rho_{B C}$ is,

$$
E_{F}\left(\rho_{B C}\right)=\left\{\begin{array}{l}
0, F \in \mathrm{I} \\
H_{2}(\gamma(F))+(1-\gamma(F)) \log (d-1), \\
F \in \mathrm{II} \\
\frac{d \log (d-1)}{d-2}(F-1)+\log d, F \in \mathrm{III}
\end{array}\right.
$$

where cases I,II,III are $\left[0, \frac{1}{d}\right],\left[\frac{1}{d}, \frac{4(d-1)}{d^{2}}\right],\left[\frac{4(d-1)}{d^{2}}, 1\right]$, respectively, $\gamma(F)=\frac{1}{d}(\sqrt{F}+\sqrt{(d-1)(1-F)})^{2}$, $H_{2}(x)=-x \log _{2} x-(1-x) \log _{2}(1-x)$.

In Fig.2, we plot $I\left(\rho_{B C}\right)-2 E_{F}\left(\rho_{B C}\right)$ for $d=$ $4,5,10,15,115$, whose lines are arranged from right to left. It can be seen that quantum discord is monogamous when $F$ is small which means $\rho_{B C}$ has less singlet fractions. With the increasing of dimension $d$, singlet $\left|\Psi^{+}\right\rangle$has higher proportion and the quantum discord has larger polygamous region. These results are consistent with the results obtained for generalized pure three-qubit GHZ and W states. 
Completely similar analysis can be made on the following Werner states,

$$
w_{B C}(x)=\frac{d-x}{d^{3}-d} I+\frac{d x-1}{d^{3}-d} P, x \in[-1,1]
$$

where $P=\sum_{i, j=1}^{d}|i j\rangle\langle j i|$ is the flip operator. We note that these analysis has been carried out in [33] and the results are quite similar with isotropic states given above.

Observation based on squashed entanglement.-For a pure tripartite state $\left|\Psi_{A B C}\right\rangle$, if its reduced bipartite state $\rho_{B C}$ satisfies $E_{D}\left(\rho_{B C}\right)=E_{F}\left(\rho_{B C}\right)$, then the monogamy relations (26) hold, where $E_{D}\left(\rho_{B C}\right)$ is the entanglement of distillation. This observation can be proved with squashed entanglement which is defined in terms of conditional mutual information 35],

$E_{s q}\left(\rho_{B C}\right):=\inf \left\{\frac{1}{2} I(B, C \mid E): \rho_{B C E}\right.$ extension of $\left.\rho_{B C}\right\}$.

In [35], it has been proved that $E_{D} \leq E_{s q}\left(\rho_{B C}\right)$. Meanwhile we have $E_{s q}\left(\rho_{B C}\right) \leq \frac{1}{2} I\left(\rho_{B C}\right)$ since $I(B, C \mid E)$ is the "squashed" correlation from $I\left(\rho_{B C}\right)$ where the classical correlations are squashed out as much as possible. Obviously, when $E_{D}=E_{F}$, inequality (6) is satisfied and quantum discord monogamy relation (2) between $A$ and $B, C$ in $\left|\Psi_{A B C}\right\rangle$ holds.

\section{UPPER BOUNDS ON QUANTUM DISCORD AND CLASSICAL CORRELATIONS}

In Ref. [36], it was conjectured that, given a bipartite sate $\rho_{A B}$ defined in the Hilbert space $H_{A} \otimes H_{B}$, the following upper bounds for quantum discord and classical correlations could exist:

$$
\begin{aligned}
J^{\rightarrow}\left(\rho_{A B}\right) & \leq \min \left[S\left(\rho_{A}\right), S\left(\rho_{B}\right)\right], \\
D^{\rightarrow}\left(\rho_{A B}\right) & \leq \min \left[S\left(\rho_{A}\right), S\left(\rho_{B}\right)\right],
\end{aligned}
$$

In [37], the upper bound of classical correlation (12) is proved to be true and the upper bound of quantum correlation (13) is proved to be true only under some conditions. In [28], based on known results of three qubits [19], Giorgi showed that the above two upper bounds hold for rank-2 states of two qubits. Now, with the features of quantum discord, we provide some more concise discussions on these upper bounds. First, we assume the mixed $\rho_{A B}$ to be reduced from a pure tripartite state $\left|\phi_{A B C}\right\rangle$ such that $\operatorname{Tr}_{C}\left|\phi_{A B C}\right\rangle\left\langle\phi_{A B C}\right|=\rho_{A B}$.

Upper bound on classical correlation.-According to its definition, the classical correlation is $J^{\rightarrow}\left(\rho_{A B}\right)=$ $S\left(\rho_{B}\right)-\min S\left(B \mid\left\{E_{j}^{A}\right\}\right)=S\left(\rho_{B}\right)-E_{F}\left(\rho_{B C}\right) \leq S\left(\rho_{B}\right)$, the last inequality comes from the fact $E_{F}(B C) \geq 0$. In order to show that we simultaneously have $J^{\rightarrow} \leq S\left(\rho_{A}\right)$, we need to prove the following inequality,

$$
E_{F}\left(\rho_{B C}\right) \geq S\left(\rho_{B}\right)-S\left(\rho_{B C}\right) .
$$

This obviously holds since $S\left(\rho_{B}\right)-S\left(\rho_{B C}\right)$ is the coherent information [38, 39] which is a lower bound for distillable entanglement smaller than EoF. Therefore we know that the upper bounds for classical correlation in (12) holds.

Upper bound on quantum correlation.-Here the quantum correlation measure is just quantum discord, it is $D^{\rightarrow}\left(\rho_{A B}\right)=S\left(\rho_{A}\right)-S\left(\rho_{A B}\right)+\min S\left(B \mid\left\{E_{j}^{A}\right\}\right)=S\left(\rho_{A}\right)-$ $S\left(\rho_{C}\right)+E_{F}\left(\rho_{B C}\right)=S\left(\rho_{A}\right)-S\left(\rho_{C}\right)+\min S\left(C \mid\left\{E_{j}^{A}\right\}\right)=$ $S\left(\rho_{A}\right)-J^{\rightarrow}\left(\rho_{A C}\right) \leq S\left(\rho_{A}\right)$, the last inequality comes from the fact that $J \rightarrow\left(\rho_{A C}\right) \geq 0$ or the concavity of entropy if we consider that $S\left(\rho_{C}\right)-E_{F}\left(\rho_{B C}\right) \geq 0$. With one half of inequality (13) proved, can we simultaneously prove another half of the inequality, $D^{\rightarrow}\left(\rho_{A B}\right) \leq S\left(\rho_{B}\right)$ ? We only need to consider the case $S\left(\rho_{A}\right)>S\left(\rho_{B}\right)$, then we should have,

$$
E_{F}\left(\rho_{B C}\right) \leq S\left(\rho_{B}\right)+S\left(\rho_{A B}\right)-S\left(\rho_{A}\right),
$$

Since $\left|\phi_{A B C}\right\rangle$ is a pure state, it is equivalent to

$$
E_{F}\left(\rho_{B C}\right) \leq I\left(\rho_{B C}\right) .
$$

In general we consider that mutual information quantifies the total correlations, it naturally seems to be larger than EoF which only quantifies quantum correlation. However this is not true! Hayden, Leung and Winter [40] found that EoF in a bipartite state can be larger than its mutual information. In [33], Li and Luo consolidated their findings and showed that Werner state [41] has this property. From a Werner state $\rho_{B C}$ (10) which violates (16), a purified tripartite state $\left|\phi_{A B C}\right\rangle$ can be constructed with $S\left(\rho_{A}\right)>S\left(\rho_{B}\right)$. Then its reduced bipartite state $\rho_{A B}$ violates the upper bound in (13). On the other hand, the violation of this upper bound can be understood from the viewpoint of coherent information. Since $E_{F}\left(\rho_{B C}\right)+J^{\rightarrow}\left(\rho_{A B}\right)=S\left(\rho_{B}\right)$ [23], inequality (15) is equivalent to

$$
S\left(\rho_{A}\right)-S\left(\rho_{A B}\right) \leq J^{\rightarrow}\left(\rho_{A B}\right),
$$

where $S\left(\rho_{A}\right)-S\left(\rho_{A B}\right)$ is one-way coherent information with classical communication from $B$ to $A$ and $J \rightarrow\left(\rho_{A B}\right)$ is one-way distillable common randomness with classical communication from $A$ to $B$, see Ref. 23]. We know that coherent information is a lower bound for distillable entanglement which is a lower bound for secret key, while the secret key rate between $A$ and $B$ is obviously smaller than their distillable common randomness. Therefore the violation of (17) means that there are states from which the distillable secret key with classical communication from $B$ to $A$ can even be larger than the distillable common randomness with classical communication from $A$ to $B$.

Thus in general the conjectured upper bound of quantum discord in relation (13) does not hold, however, a released upper bound of quantum discord can be obtained,

$$
D^{\rightarrow}\left(\rho_{A B}\right) \leq \max \left[S\left(\rho_{A}\right), S\left(\rho_{B}\right)\right] .
$$

\section{CONCLUSION}

Quantum discord is an important quantum correlation measure. In this paper, we discuss a monogamy relation 
for this measure which is different from the monogamy relation proposed in Refs. 27, 28]. For a tripartite pure state, monogamy relation (2) is reduced to a relation (6) between mutual information and entanglement of formation in a reduced bipartite state. Since relation (6) does not generally hold, monogamy relation (2) can be both monogamous and polygamous for arbitrary pure tripartite states. Through numerical calculations with several explicit classes of states, we show that monogamy relation (2) is roughly related to the entangled proportion shared in the reduced bipartite state. It holds when the entangled proportion is small and turns into a polygamy relation when the entangled proportion is large.

In this article, we also provide a concise discussion on a conjecture of upper bounds for classical and quantum correlations in a bipartite state [10]. We show that the upper bounds on quantum correlation may be violated with a pure tripartite state constructed from a Werner state. The physical meaning behind the violation is discussed with operational quantum information concepts. At the same time, a released upper bound (18) still holds. Our results should be useful for further understanding of quantum discord and distribution of classical and quantum correlations in multipartite states.

Acknowledgments. - We thank Shuai Cui for useful discussions. This work is supported by "973" program (2010CB922904) and NSFC (10974247, 11175248, U1204114, 11047174). X-J Ren acknowledges financial support from the education department of Henan province.
[1] R. Horodecki, P. Horodecki, M. Horodecki and K. Horodecki, Rev. Mod. Phys. 81, 865 (2009).

[2] R. F. Werner, Phys. Rev. A 40, 4277 (1989).

[3] E. Knill and R. Laflamme, Phys. Rev. Lett. 81, 5672 (1998).

[4] L. Henderson and V. Vedral, J. Phys. A: Math. Gen. 34, 6899 (2001).

[5] H. Ollivier and W. H. Zurek, Phys. Rev. Lett. 88, 017901 (2001).

[6] J. Cui and H. Fan, J. Phys. A: Math. and Theor. 43, 045305 (2010).

[7] K. Modi, T. Paterek, W. Son, V. Vedral and M. Williamson, Phys. Rev. Lett. 104, 080501 (2010).

[8] M. Piani, S. Gharibian, G. Adesso, J. Calsamiglia, P. Horodecki, and A. Winter, Phys. Rev. Lett. 106, 220403 (2011).

[9] A. Streltsov, H. Kampermann, and D. Bruss, Phys. Rev. Lett. 106, 160401 (2011).

[10] S. Luo and S. Fu, Phys. Rev. Lett. 106, 120401 (2011).

[11] A. Datta, A. Shaji, and C. M. Caves, Phys. Rev. Lett. 100, 050502 (2008).

[12] P. Giorda and Matteo G. A. Paris, Phys. Rev. Lett. 105, 020503 (2010); G. Adesso and A. Datta, ibid 105, 030501 (2010)

[13] B. Dakić, V. Vedral and Časlav Brukner, Phys. Rev. Lett. 105, 190502 (2010).

[14] A. Shabani and Daniel A. Lidar, Phys. Rev. Lett. 102, 100402 (2009); C. A. Rodriguez-Rosario, G. Kimura, H. Imai, and A. Aspuru-Guzik, Phys. Rev. Lett. 106, 050403 (2011).

[15] D. Cavalcanti, L. Aolita, S. Boixo, K. Modi, M. Piani and A. Winter, Phys. Rev. A 83, 032324 (2011); V. Madhok and A. Datta, ibid 83, 032323 (2011).

[16] R. Auccaise, L. C. Celeri, D. O. Soares-Pinto, E. R. deAzevedo, J. Maziero, A. M. Souza, T. J. Bonagamba, R. S. Sarthour, I. S. Oliveira, and R. M. Serra, Phys. Rev. Lett. 107, 140403 (2011).

[17] A. Streltsov, H. Kampermann, and D. Bruss, Phys. Rev. Lett. 107, 170502 (2011).

[18] R. Auccaise, J. Maziero, L. C. Celeri, D. O. Soares-Pinto, E. R. deAzevedo, T. J. Bonagamba, R. S. Sarthour, I. S. Oliveira, and R. M. Serra, Phys. Rev. Lett. 107, 070501 (2011).
[19] V. Coffman, J. Kundu and W. K. Wootters, Phys. Rev. A 61, 052306 (2000).

[20] T. J. Osborne and F. Verstraete, Phys. Rev. Lett. 96, 220503 (2006).

[21] S. Hill and W. K. Wootters, Phys. Rev. Lett. 78, 5022 (1997); W. K. Wootters, Phys. Rev. Lett. 80, 2245 (1998).

[22] T. Hiroshima, G. Adesso and F. Illuminati, Phys. Rev. Lett. 98, 050503 (2007); G. Adesso and F. Illuminati, ibid 99, 150501 (2007).

[23] M. Koashi and A. Winter, Phys. Rev. A 69, 022309 (2004).

[24] M. Seevinck, Quantum Information Processing, vol. 9, 273-294 (2010); A. Kay, D. Kaszlikowski, and R. Ramanathan, Phys. Rev. Lett. 103, 050501 (2009); M. Pawłowski and Č. Brukner, ibid 102, 030403 (2009); P. Kurzyński, T. Paterek, R. Ramanathan, W. Laskowski, and D. Kaszlikowski, ibid 106, 180402 (2011)

[25] Y. C. Ou and H. Fan, Phys. Rev. A 75, 062308 (2007).

[26] Y. C. Ou, H. Fan, and S. M. Fei, Phys. Rev. A 78, 012311 (2008).

[27] R. Prabhu, A. K. Pati, A. Sen(De), and U. Sen, arxiv: 1108.5168, (2011).

[28] G. L. Giorgi, Phys. Rev. A 84, 054301 (2011).

[29] C. H. Bennett, D. P. DiVincenzo, J. A. Smolin, and W. K. Wootters, Phys. Rev. A 54, 3824 (1996); M. Horodecki, Quantum Inf. Comput. 1, 3 (2001); W. K. Wootters, Quantum Inf. Comput. 1, 27 (2001).

[30] F. F. Fanchini, M. F. Cornelio, M. C. de Oliveira and A. O. Caldeira, Phys. Rev. A 84, 012313 (2011).

[31] F. F. Fanchini, M. C. de Oliveira, L. K. Castelano and M. F. Cornelio, arXiv:1110.1054

[32] B. Groisman, S. Popescu, and A. Winter, Phys. Rev. A 72, 032317 (2005).

[33] N. Li and S. Luo, Phys. Rev. A 76, 032327 (2007).

[34] B. M. Terhal and K. G. H. Vollbrecht, Phys. Rev. Lett. 85,2625 (2000).

[35] M. Christandl, and A. Winter, J. Math. Phys, 45, 829 (2003).

[36] S. Luo, S. Fu, and N. Li, Phys. Rev. A 82, 052122 (2010).

[37] L. Zhang and J. Wu, arXiv:1105.2993

[38] P. Horodecki, R. Horodecki, and M. Horodecki, arxiv:quant-ph/9805072 
[39] C. H. Bennett et al., Phys. Rev. A 54, 3824 (1996); I. Devetak and A. Winter, Phys. Rev. Lett. 93, 080501 (2004).

[40] P. Hayden, D.W. Leung, and A.Winter, Commun. Math. Phys. 265, 95 (2006).
[41] K. G. H. Vollbrecht and R. F. Werner, Phys. Rev. A 64, 062307 (2001). 